\title{
A clean sweep: mucin bundles clear the airway
}

\author{
Susan Birket \\ Affiliation: University of Alabama at Birmingham - Medicine, Birmingham, AL, USA.
}

Correspondence: Susan Birket, University of Alabama at Birmingham - Medicine, 1918 University Blvd MCLM 794, Birmingham, AL 35294, USA. E-mail: susanbirketवuabmc.edu

@ERSpublications

Mucus bundles are important for airway clearance and are regulated by cholinergic stimulus http://ow.ly/Hovc30liwNo

Cite this article as: Birket S. A clean sweep: mucin bundles clear the airway. Eur Respir J 2018; 52: 1801144 [https://doi.org/10.1183/13993003.01144-2018].

\begin{abstract}
Mucostasis and small airway plugging lead to chronic infection, hyperinflammation, and a spiral of progressive lung damage in cystic fibrosis (CF), ultimately causing bronchiectasis and pulmonary function decline [1]. Even in the absence of bacterial infection, mucus accumulation can lead to inflammatory upregulation, resulting in lung damage despite bacterial eradication [2]. Airway mucus has been postulated to be overproduced and inefficiently transported in CF $[3,4]$. In human epithelial cells as well as multiple animal models of CF, mucus is hyperviscous and abnormally elastic [5-9]. In CF pigs, mucus is also aberrantly tethered to the gland duct openings, a factor which may underlie the propensity for CF mucus to spend less time in motion than in the normal condition [10]. While the mucus layer of the airway surface liquid (ASL) contains numerous proteins, surfactants, antibacterial proteases and anti-proteases [11], its major components are mucins [12]. Mucins are large glycopolymers that can either be tethered to the epithelium or secreted into the lumen as gel-forming polymers, as is the case with MUC5B and MUC5AC [13]. While the roles of individual mucins, namely MUC5B and MUC5AC, have been well identified in other disease states, such as asthma and bacterial infections, it is less well delineated how they are overproduced or dysregulated in CF [13]. Furthermore, in CF, in the absence of adequate bicarbonate concentrations, mucins may not exist in the appropriate conformation, a potential cause of many of these abnormalities $[14,15]$. Yet, despite all that is known about mucins and mucus in CF and other muco-obstructive diseases, a means to correcting these abnormalities and restoring normal mucus clearance has yet to be identified.
\end{abstract}

Despite an incomplete understanding of the detailed mechanisms which drive the mucociliary clearance apparatus, airway clearance has been a therapeutic target for disease states such as CF for decades. Inhaled therapies such as Pulmozyme and hypertonic saline have been in use as mucolytics [16, 17], with new agents under development for the same purpose [18, 19], not just for CF but for other muco-obstructive diseases [20]. However, this has been challenging, probably based on the relative lack of knowledge by which native mucus is cleared from the airway, and how these mechanisms are disrupted in disease. Identification of an innate mechanism by which the airway clears mucus underscores the importance of this as a therapeutic option.

The article by ERmund et al. [21] in this issue of the European Respiratory Journal uses normal and CF pig tracheobronchial explants to examine in detail the mucin bundles responsible for sweeping the airway clean, the pharmacological mechanisms under which this process is amplified or inhibited, and the ways by which this process becomes aberrant in disease. Importantly, these data help to make the distinction 
between the ASL and bundles of mucin, which appear to transport independently, at different rates, and respond separately to cholinergic stimulus.

ERMUND et al. [21] showed distinct strands of mucus, which they have termed bundles, both emerging from glands and moving across the airway surface in the normal and CF piglet trachea. These appear to be comprised of MUC5B at the core, with a coating of MUC5AC, and sweep along the surface of the trachea. While these bundles exhibited contact points with goblet cells in both the wild-type and CF tracheae, the bundles are closer, and appear to have more points of attachment in CF. Excess contact with goblet cells in the epithelial layer is likely contributing to the delayed movement of the bundles. Importantly, the bundles are moving separately from beads that are associated with the ASL; these are moving at faster rates and increase their speed following cholinergic stimulus. The bundles respond oppositely to cholinergic agents; while the addition of carbachol increased velocity of the beads in the ASL, it simultaneously decreased the velocity of the bundles. The effect was the same in the CF trachea, although baseline speeds of both bundles and particles associated with the ASL were moving at much slower rates than in the wild-type. The hypothesis that the bundles are cleaning the airway was demonstrated with the addition of Pseudomonas aeruginosa to the trachea. In WT pigs, bacteria were quickly bound to the mucin bundles and cleared from the airway surface. By contrast, bacteria added to CF pig tracheae were localised to both the bundles and the epithelial surface. Retention of the bundles allowed for retention of the bacteria in the CF condition.

Interestingly, in the normal trachea the inhibitory effect of carbachol on mucin bundle velocity was reversible. In the presence of carbachol, mucus bundles in the normal pig trachea were slowed dramatically. When the normal trachea was pre-treated with ipratropium bromide, and thereby subjected to anticholinergic effects, mucin bundles exhibited some restoration of velocity, although they did not reach pre-carbachol speeds, and the effect was temporary; after $45 \mathrm{~min}$ the movement of the bundles had slowed again. In the CF condition, however, pre-treatment with ipratropium did not increase mucin bundle velocity, suggesting that blocking the cholinergic pathway cannot compensate for the other differences noted in the CF mucin bundles, such as increased contact with the epithelium.

The novel finding that cholinergic stimulus has separate and opposite effects on the ASL and the mucin bundles is important, and may have significant effects on novel therapeutics. While the hydration and ionic composition of the ASL is indeed important, it remains to be answered whether correction of these deficiencies in disease states such as CF or chronic obstructive pulmonary disease (COPD) will affect the mucin bundles, i.e. by decreasing excess contact with the epithelium to restore transport along the airway surface, and, consequently, increase bacterial clearance. Given the likely abundance of cholinergic stimulus in muco-obstructive disease states, as the host attempts to clear excess airway irritants by increasing gland secretions and ciliary beating, anti-cholinergic therapies may have some use [22]. While untested in a COPD disease model, the hypothesis that ipratropium bromide may benefit this patient population by increasing clearance of mucin bundles from the airway suggests a more complicated picture of mucus clearance than originally thought. While the effects of ipratropium bromide on transport in the ASL compartment were untested, and the transport-restoration phenomenon did not occur in the CF trachea, this warrants future investigation. The benefits of anticholinergic therapy to target mucus clearance, while on the surface counter-intuitive, may be targetable to specific states of disease.

The authors posit that slowing the mucin bundles in response to cholinergic stimulus allows for capture of bacteria for the purpose of clearance from the airway. While this physiological mechanism may be effective in the normal lung, slowing of the mucus bundles in CF may result in abnormal attachment to the epithelial goblet cells, and could be a target for pharmacological correction. This mechanism may also highlight the difference between mucus and the mucin compartment inside the mucus, and point to a need for more detailed analysis, and more targeted therapeutics.

Conflict of interest: S. Birket has nothing to disclose.

\section{References}

Ratjen F, Doring G. Cystic fibrosis. Lancet 2003; 361: 681-689.

2 Rosen BH, Evans TIA, Moll SR, et al. Infection is not required for mucoinflammatory lung disease in CFTR-knockout ferrets. Am J Respir Crit Care Med 2018; 197: 1308-1318.

3 Hoegger MJ, Awadalla M, Namati E, et al. Assessing mucociliary transport of single particles in vivo shows variable speed and preference for the ventral trachea in newborn pigs. Proc Natl Acad Sci USA 2014; 111: 2355-2360.

4 Birket SE, Chu KK, Liu L, et al. A functional anatomic defect of the cystic fibrosis airway. Am J Respir Crit Care Med 2014; 190: 421-432.

5 Birket SE, Chu KK, Houser GH, et al. Combination therapy with cystic fibrosis transmembrane conductance regulator modulators augment the airway functional microanatomy. Am J Physiol Lung Cell Mol Physiol 2016; 310: L928-L939. 
6 Birket SE, Davis JM, Fernandez CM, et al. Development of an airway mucus defect in the cystic fibrosis rat. JCI Insight 2018; 3: 97199.

7 Derichs N, Jin BJ, Song Y, et al. Hyperviscous airway periciliary and mucous liquid layers in cystic fibrosis measured by confocal fluorescence photobleaching. Faseb J 2011; 25: 2325-2332.

8 Broughton-Head VJ, Shur J, Carroll MP, et al. Unfractionated heparin reduces the elasticity of sputum from patients with cystic fibrosis. Am J Physiol Lung Cell Mol Physiol 2007; 293: L1240-L1249.

9 Dawson M, Wirtz D, Hanes J. Enhanced viscoelasticity of human cystic fibrotic sputum correlates with increasing microheterogeneity in particle transport. J Biol Chem 2003; 278: 50393-50401.

10 Hoegger MJ, Fischer AJ, McMenimen JD, et al. Impaired mucus detachment disrupts mucociliary transport in a piglet model of cystic fibrosis. Science 2014; 345: 818-822.

11 Evans TI, Joo NS, Keiser NW, et al. Glandular proteome identifies antiprotease cystatin C as a critical modulator of airway hydration and clearance. Am J Respir Cell Mol Biol 2016; 54: 469-481.

12 Button B, Cai LH, Ehre C, et al. A periciliary brush promotes the lung health by separating the mucus layer from airway epithelia. Science 2012; 337: 937-941.

13 Rose MC, Voynow JA. Respiratory tract mucin genes and mucin glycoproteins in health and disease. Physiol Rev 2006; 86: 245-278.

14 Kesimer M, Makhov AM, Griffith JD, et al. Unpacking a gel-forming mucin: a view of MUC5B organization after granular release. Am J Physiol Lung Cell Mol Physiol 2010; 298: L15-L22.

15 Yang N, Garcia MA, Quinton PM. Normal mucus formation requires cAMP-dependent $\mathrm{HCO}_{3}^{-}$secretion and $\mathrm{Ca}^{2+}$. mediated mucin exocytosis. J Physiol (Lond) 2013; 591: 4581-4593.

16 Flume PA, O'Sullivan BP, Robinson KA, et al. Cystic fibrosis pulmonary guidelines: chronic medications for maintenance of lung health. Am J Respir Crit Care Med 2007; 176: 957-969.

17 Goralski JL, Wu D, Thelin WR, et al. The in vitro effect of nebulised hypertonic saline on human bronchial epithelium. Eur Respir J 2018; 51: 1702652.

18 Ermund A, Recktenwald CV, Skjak-Braek G, et al. OligoG CF-5/20 normalizes cystic fibrosis mucus by chelating calcium. Clin Exp Pharmacol Physiol 2017; 44: 639-647.

19 Middleton A, Robinson PD, McKay K, et al. A pilot study of inhaled dry-powder mannitol during cystic fibrosis-related pulmonary exacerbation. Eur Respir J 2015; 45: 541-544.

20 Aaron SD. Mucolytics for COPD: negotiating a slippery slope towards proof of efficacy. Eur Respir J 2017; 50: 1701465.

21 Ermund A, Meiss LN, Dolan B, et al. The mucin bundles responsible for airway cleaning are retained in cystic fibrosis and by cholinergic stimulation. Eur Respir J 2018; 52: 1800457.

22 Widdicombe JH, Wine JJ. Airway Gland Structure and Function. Physiol Rev 2015; 95: 1241-1319. 